\title{
A Light Weight Name Service and its use within a collaborative editor*
}

\author{
J.-C. Lugeon \\ Département d'Informatique. \\ F. Pacull \\ Ecole Polytechnique Fédérale de Lausanne \\ CH-1015 Lausanne (Switzerland) \\ E-mail: $\{$ lugeon,pacull\}@lse.epfl.ch
}

\begin{abstract}
This paper presents the LWNS, a light weight name service specifically designed for groupware applications, and an example of its use. The chosen application, called DUPLEX, is a collaborative editing environment for users connected through the Internet. It proposes a model based on splitting the document into independent parts, maintained individually and replicated within a distributed kernel. Naming is an important aspect of such application since objects (document parts) are replicated and distributed over the large heterogeneous network. However, this set is small (typically a few dozen elements) and composed of objects whose names are contextual to the document. The replicas are maintained in heterogeneous file systems with no global naming scheme consistent with the name space of the collaboration. Therefore it requires a dedicated name service specifically designed to solve this problem in large scale distributed applications.
\end{abstract}

\section{Keywords}

Naming, large scale distributed systems, CSCW

\section{INTRODUCTION}

Distributed applications such as a collaborative editing environment use the network not only to exchange information, but also to share objects containing data. Sharing objects, in general implies that the users involved should have some knowledge about the objects: its name, generally given in a user friendly manner, but also the method used to access it. This second

*Research supported by the FNS and OFES under contract number 21-32210.91, as part of European ESPRIT Basic Research Project Number 6360 (BROADCAST). 
component is generally related to a more computer-oriented pattern. The complexity of this pattern in linked to the complexity of the considered object and the underlying distributed system.

For instance, consider that objects are files. When all users work in the same integrated file system domain (e.g. NFS (Sandberg et al., 1985) or AFS (Howard et al., 1988)), the object reference is the path name, i.e. any composite path, the user is authorized to follow leading from the root of the shared file name space to the file. When the users and the resources reside in heterogeneous systems, access to the objects requires a more complicated protocol (e.g. file transfer service as FTP (Postel and Reynolds, 1985)). In addition to the the file name relative to this server, the server's address and the protocol (here FTP) are required.

When the object is replicated and spread over a large heterogeneous network, the object's pattern becomes more and more complex. It could include the list of the replicas, the protocol used for ensuring consistency or the address of a replicated service. Thus, the applications has to rely on a dedicated service that ensures the translation between the object name and the means to access it. This service has to ensure the following requirements:

- To name objects of any type, e.g. files and services.

- To provide name transparency, i.e. a name must not contain any information specific to the underlying system, and a name must refer to an object independently of the site where the user issue the requests.

- To be flexible, i.e. the name service should adapt itself to the requirements of the application and not the contrary.

A Light Weight Name Service (noted LWNS) has been developed in our laboratory to fulfill these requirements for applications considering a large scale distributed system like the Internet. This paper describes an experience of using the LWNS in a collaborative editing environment called DUPLEX (Pacull et al., 1994; Pacull, 1995) also developed in our laboratory.

The paper is structured as follows: Section 2 presents briefly the motivation that has conducted to the conception of the LWNS. The LWNS model is described in Section 3. Section 4 gives an overview of the DUPLEX collaborative editing environment and emphasizes the problems related to naming. Section 5 describes how the LWNS has been used to this end. Section 6 presents why existing name services cannot be used in the particular frame of collaborative application over a large scale distributed system. Finally, Section 7 concludes the paper.

\section{MOTIVATION}

The distributed system we consider is composed of a set of sites in a wide area network. We consider only the sites involved within a collaboration. Sites for different collaborations can be disjoint or overlap, but are managed as independent distributed systems so large scale does not necessarily imply a large number of sites. Large scale distributed system for the particular frame considered here means large distances between sites, since they might be scattered over continents and connected through the Internet (Comer, 1988).

For instance, if we consider collaborative editing, a site could be the workstation of an author, a data repository for parts of the document, or both. The set of sites evolves dynamically as users join or leave the collaboration, and parts of a document are added or removed. 
Considering such a distributed system, the constraints of the large scale and the specificity of the collaborative application, should be taken into account.

The first point implies that failures (links or sites) have to be considered. In particular, two sites might be unable to communicate, though both can communicate with a common destination.

The second point has an influence on the naming aspect since the information useful to the collaboration is distributed over the large underlying heterogeneous system. The closure of the name spaces of all these heterogeneous systems is very wide, and considering naming within this context is costly even if the set of objects (information about the collaboration) is relatively small. Moreover, the names of the objects are contextual to the collaboration and do not correspond to the naming scheme of the underlying heterogeneous systems.

Our approach is to provide a dedicated name service (1) that is not dependent on the architecture of the underlying system, and (2) that ensures the availability of the service despite failures or network partitions.

\section{THE LIGHT WEIGHT NAME SERVICE}

This Section presents a context-relative name service, called LWNS for Light Weight Name Service, specifically designed for large scale distributed applications.

\subsection{LWNS basic model}

The LWNS manages the mapping of contextual names, meaningful for the users in the frame of a collaborative application, to information. By "information", we mean all that the users want to be associated with the contextual name.

The LWNS is a context-relative name service distributed over several name servers, where each name server is responsible for an independent part of the global name space. Therefore, the unavailability of one of the name servers (failure or network partition) does not have any harmful effects on the other name servers, i.e. users will only notice the failure if they need to access contextual names managed by the unavailable name server. Moreover, instead of other context-relative name services such as in ANSA (Linden, 1993), the naming is global to the collaboration and not context-relative to each name server.

The LWNS furnishes only basic operations (thus the term light weight) such as: to register a name and some information, unregister this information and look up a name to retrieve the associated information. Despite of this very simple interface and functionality, we claim that it furnishes the minimal required for many collaborative applications. The advantage of this simplicity is that the name service can be realized at a low cost. More complex features, if they are required, can be added on top of it (e.g. at the application level).

Each name server manages two contexts, a shared context and a private context. The shared context is exported to the collaboration, whereas the private context is only accessible by requesting directly the name server and permits to cache some information (Sollins and Clark, 1988). As will be described in the following, the cached information is used (1) for accelerating name resolution, and (2) in case of failures of parts of the name service. 


\section{Object references}

In general terms, accessing an object requires the following knowledge:

- the address of a service providing the access to the object, e.g. for remote access to a file, a service of the resident file system which can be invoked through the network.

- an access protocol, e.g. NFS, FTP, HTTP, ...

- the object's local name, i.e. the name of the object for the considered couple (address of service, protocol).

This information defines what we call the object reference. An object reference is associated to the contextual name manipulated by the users and the LWNS is in charge of the mapping between the contextual name and the object reference.

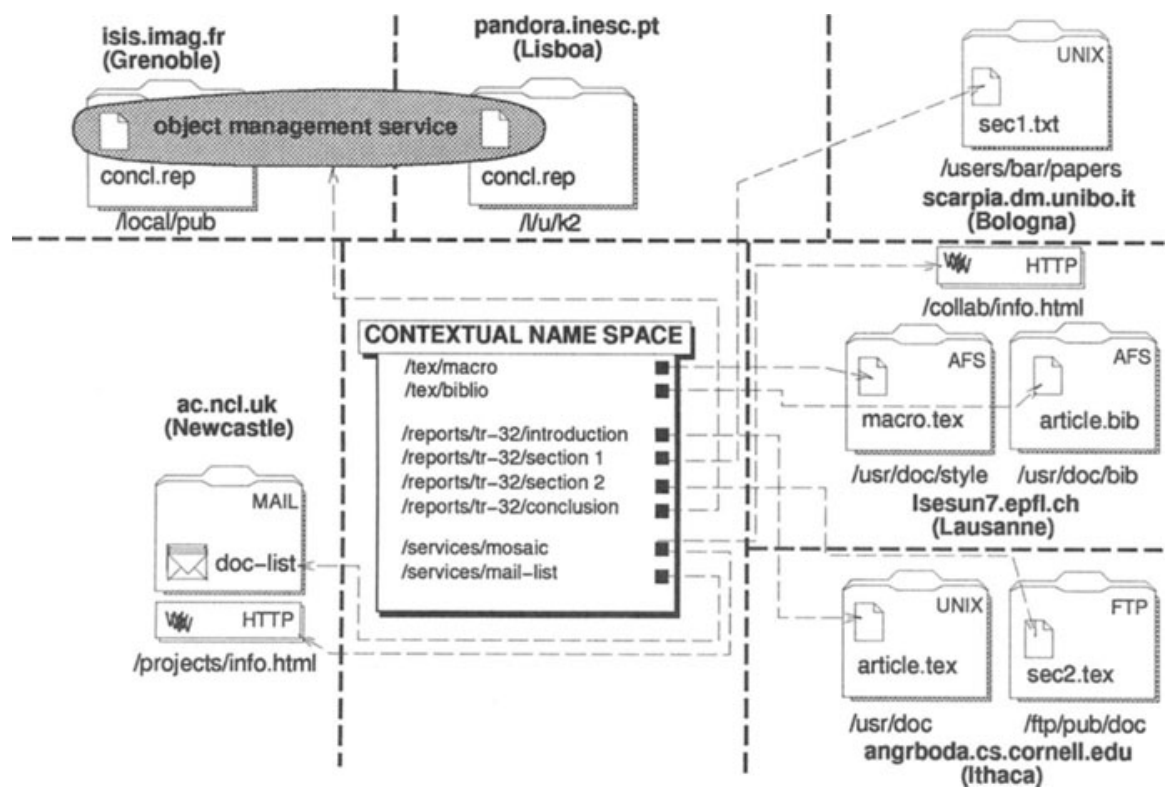

Figure 1 Contextual name space with the object references mapping.

Figure 1 shows the mapping between a set of contextual names defined within the frame of a collaboration and the corresponding object references. An object reference is represented by a black square pointing to the object location.

An object can be a traditional file, maintained by a file system and accessible via the FTP protocol (e.g. the contextual name /reports/tr-32/section2 corresponds to the file $/ \mathrm{ftp} / \mathrm{pub} / \mathrm{doc} / \mathrm{sec} 2$.tex on site angrboda.cs.cornell.edu). Other services of higher or lower complexity can also be considered. The service can be a mailing list maintained 
by one site, e.g. ac. ncl.uk, or two identical services, e.g. HTTP which can be offered either by ac. ncl.uk or lsesun7.epfl. ch. In this last case, the service is replicated but each server is independent. Finally, it is possible to consider a service maintaining a replicated object, /reports/tr-32/conclusion, which is maintained by a replicated service on two sites (pandora.inesc.pt and isis.imag.fr). The service in this case can be represented by the list of the involved sites or by a group name if a more complex paradigm is used for managing the replication. Note that the contextual name is completely independent from the way that the object is actually implemented.

\subsection{Architecture of the LWNS}

The name service is distributed over several independent name servers. We note LWNS $_{s_{i}}$ the server located on site $s_{i}$. Each $\mathrm{LWNS}_{s_{i}}$ is independent of the others and only manages a subset of the references of the objects available in the system (i.e. a context). Depending on the properties that we require for the name service (e.g. availability, locality, etc.), the context managed by a LWNS $_{s_{i}}$ can be one of the following:

1. the contextual names corresponding to the objects located on $s_{i}$. This allows to enforce availability by ensuring that if a site $s_{i}$ is up then the object can actually be accessed, since the LWNS $_{s_{i}}$ and the service managing the object on site $s_{i}$ are reachable. If the two services were on different sites, the failure of one of the two sites would be sufficient to make the object inaccessible.

2. the contextual names corresponding to the objects which have been created by the site $s_{i}$. This does not mean that they are located on $s_{i}$ but only that the site $s_{i}$ had asked for the creation. This solution enforces locality for the object creation. This point will be discussed in Section 5 as it is the option used by DUPLEX.

3. any subset of the contextual name space providing that the whole contextual name space is distributed over the different LWNS $_{s_{j}}$.

This constitutes a flexibility, which is particularly interesting as it allows to adapt the LWNS to the requirements of the application.

Since each LWNS $s_{i}$ is responsible for the management of a part of the global context, they should be grouped together in order to form a federation. This is realized thanks to a master server (noted LWNS ${ }_{m}$ ) that has two roles: (1) it is used for federating the different LWNS $_{s_{i}}$ involved within a collaboration and can therefore be compared with a Trader (Deschrevel, 1993), and (2) it is used for accelerating the name resolution by maintaining a prefix table as in Sprite (Welch and Ousterhout, 1986) (name resolution is discussed in the next Section).

The location of the $\mathrm{LWNS}_{m}$ is decided during the initial phase of the collaboration by the community that wants to collaborate; it is statically known. Upon its creation, a LWNS $_{s_{i}}$ registers itself at the level of the $\mathrm{LWNS}_{m}$, and obtains the list of the previously registered LWNS $_{s_{j}}$ as a result. Moreover, the LWNS ${ }_{m}$ forwards a notification of the existence of the new LWNS $_{s_{i}}$ to all the registered LWNS ${ }_{s_{j}}{ }^{*}$.

As a result, each LWNS $s_{i}$ knows all the other LWNS $_{s_{j}}$ registered; this information is used, as described in the next Section, for the name resolution.

*This communication as every communication within LWNS is not costly, because it uses a reliable datagram protocol ensuring good performance and reliability. 


\subsection{Name resolution}

We will now describe the means by which the name resolution is performed. Consider, that the policy is to manage the contextual names on the same site as the objects. This is illustrated in Figure 2, where the contextual name space of Figure 1 is split into six parts, corresponding to the six sites storing objects. For example, the $\mathrm{LWNS}_{\text {lsesun } 7 . e p f l . c h}$ (noted $\mathrm{LWNS}_{\text {lsesun } 7}$ in the following) only manages a name space containing the contextual names/tex/biblio, / tex/macro, and /services/mosaic, which correspond to the files/usr/doc/bib/article.bib and /usr/doc/style/macro.tex, accessible through FTP and the service HTTP. The LWNS $_{m}$ is on angrboda.cs.cornell.edu.

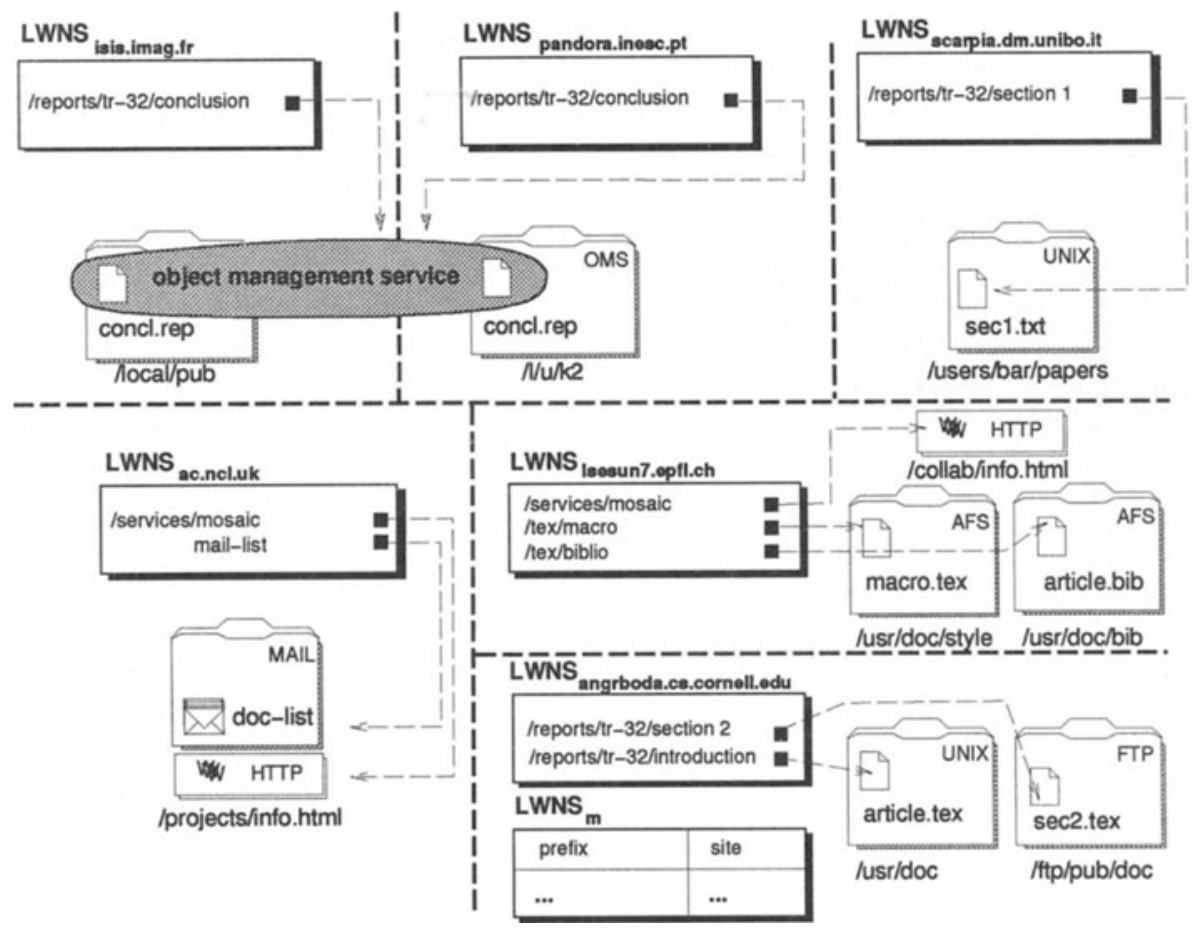

Figure 2 Name distribution based on the location of the objects.

\section{Local resolution}

The name resolution requested by the site $s_{i}$ is performed in the following way. First, the local LWNS $s_{i}$ is enquired for the $o$-ref (object reference) corresponding to a given $o$-ctxt (contextual name). If $o$-ctxt is known by the LWNS $_{s_{i}}$ then the response is immediate. For instance, if $o$-ctxt is /tex/biblio and $s_{i}$ is lsesun 7. epfl. ch then the corresponding $o$-ref

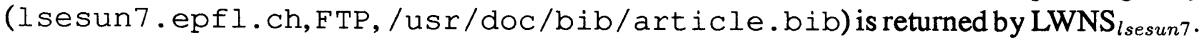




\section{Remote resolution}

In case of a negative response, a remote interrogation will be necessary. For instance, if the $o$-ctxt is the same but the requester $s_{i}$ is now isis.imag.fr. Since LWNS ${ }_{i s i s}$ does not manage the contextual name / tex/biblio it has no information on the location of the corresponding object.

There are two methods for locating the $\mathrm{LWNS}_{s_{k}}$ that manages a contextual name $o$-ctxt: (1) an efficient optimized method, and (2) a costly brute force method. The optimized method is always applied first; if it fails (due to some site crash or link failure), the costly method is then applied (see $\S$ Failures of Section 3.3).

The optimized method uses a prefix table, a technique introduced by the Sprite File System (Welch and Ousterhout, 1986), which is located at the LWNS $m$ level. The prefix table is a set of entries (prefix, site) where prefix is a prefix of a contextual name: e.g. /tex is a prefix ${ }^{*}$ for the contextual name /tex/biblio. The prefix table ensures the following property:

Consider $o$-ctxt a contextual name. If (prefix, $s_{i}$ ) is the entry of the prefix table that has the longest prefix of $o$-ctxt, then the contextual name $o$-ctxt can be resolved by the server $\mathrm{LWNS}_{s_{i}}$ located on the site $s_{i}$.

An example of a prefix table is given in Figure 3. For instance, if the $o$-ctxt is / tex/biblio, the longest corresponding prefix is /tex. The entry (/tex, lsesun7.epfl.ch) tells that all contextual names beginning by / tex (and in particular / tex/biblio can be resolved by the $\mathrm{LWNS}_{\text {lsesun } 7}$.

\begin{tabular}{|l|l|}
\hline Prefix & Site \\
\hline /service/mosaic & $\begin{array}{l}\text { Isesun7.epfl.ch } \\
\text { Itex }\end{array}$ \\
/reports/tr-32/section 1 & scarpia.dm.unibo.it \\
/reports/tr-32/introduction & angrboda.cs.cornell.edu \\
/reports/tr-32/section 2 & angrboda.cs.cornell.edu \\
/reports/tr-32/conclusion & pandora.inesc.pt \\
/reports/tr-32/conclusion & isis.imag.fr \\
/services/mail-list & ac.ncl.uk \\
/services/mosaic & ac.ncl.uk \\
\hline
\end{tabular}

Figure 3 Prefix table.

Thus, when LWNS isis $_{\text {is }}$ is asked for a name resolution that it cannot resolve locally (e.g. $o$-ctxt is /tex/biblio) the LWNS $_{m}$ is first interrogated. The result of this first request is the LWNS $_{s_{j}}$ that manages this contextual name (LWNS lsesun $7_{7}$ in our example). In a second step, LWNS $_{\text {lsesun } 7}$ is requested for the corresponding o-ref as is shown in Figure 4. 
By consulting such a prefix table, the name resolution is very efficient since it requires accessing only two servers: (1) the server managing the prefix table ( $\mathrm{LWNS}_{m}$ ), and (2) a single remote LWNS $s_{j}$ that manages the requested information.

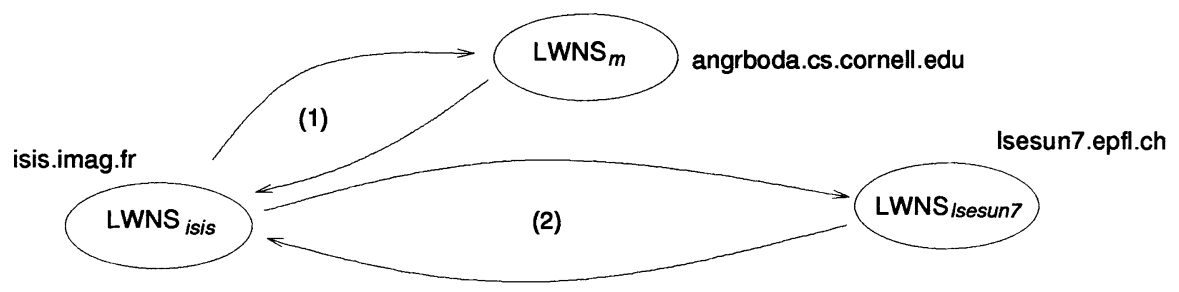

Figure 4 Remote resolution protocol.

\section{Optimization}

Each LWNS $s_{s_{i}}$ can cache information in order to make the remote name resolution more efficient. This information constitutes what we have called private context in the beginning of Section 2 . The information that is cached is (1) the result of the prefix table consultation and (2) the result of the remote $\mathrm{LWNS}_{s_{j}}$ interrogation. For instance, in our example, the information returned by the $\mathrm{LWNS}_{m}$ can be cached in order to short-cut the $\mathrm{LWNS}_{m}$ interrogation for contextual names that have the same prefix as /tex/biblio (e.g. /tex/macro). In the same manner, information returned by the $\mathrm{LWNS}_{\text {lsesun } 7}$ about / tex/biblio can be locally stored in order to permit a local resolution by the LWNS isis $_{\text {at another time. }}$

Concerning the caches, the flexibility of the LWNS permits the application designer to be free to choose the way he/she manages the information stored at the LWNS level. Thus, it is possible to register, in a $\mathrm{LWNS}_{s_{j}}$, "shared" information (prefix table updated) or "private" information (prefix table not updated). The cached information will be accessed by a local request to LWNS $s_{j}$ or by a brute force method interrogation.

By using cached information, once the resolution of a contextual name has been performed one time, the next resolution will be performed locally. Consistency can be maintained by detecting and discarding stale cache entries on use (Cheriton and Mann, 1989). Caching information is of particular interest for collaborative application, since generally the contents of a shared object can change but the reference to this object stays the same (in particular this is the case for DUPLEX).

\section{Failures}

Unfortunately, this method for the remote name resolution cannot be used when either (1) the $\mathrm{LWNS}_{m}$ or (2) the LWNS $s_{j}$ maintaining the $o$-ctxt are not accessible. In the first case because it will not be possible to find which remote LWNS $_{s_{j}}$ should be interrogated and in the second because the remote $\mathrm{LWNS}_{s_{j}}$ itself cannot be interrogated.

In both cases, the costly brute force method is applied. In this method, the local LWNS $s_{s_{i}}$ that wants to make a remote name resolution multicasts directly the request to every LWNS $_{s_{j}}$ it knows. In case (1), such a brute force method permits to reach the information since a $\mathrm{LWNS}_{s_{i}}$ 
knows all the other $\mathrm{LWNS}_{s_{j}}$ registered. In case (2), as LWNS $s_{s_{j}}$ may cache information about their previous requests, the brute force method gives a chance to find another LWNS $_{s_{k}}$ that has the information in its cache. In fact, if this object has already been accessed, the information is somewhere in a $\mathrm{LWNS}_{s_{k}}$.

We see that caching of information is not only used for accelerating requests but also in order to ensure fault tolerance by replicating information for the brute force method. This is obtained for free since the caching of information will be performed, in any case, for the acceleration goal.

As a result, the name resolution of $o$-ctxt by the site $s_{i}$ can be performed in three ways : (1) by the local LWNS $s_{i}$ if the object is managed by LWNS $_{s_{i}}$ or if the result of a previous request has been cached, (2) by the LWNS $m$ managing the prefix table and the LWNS $_{s_{j}}$ managing the name $o$-ctxt if the LWNS ${ }_{m}$ is available, or (3) by all the LWNS $s_{k}$ if the $\mathrm{LWNS}_{m}$ managing the prefix table or if the LWNS $s_{j}$ responsible for $o$-ctxt is inaccessible (e.g. due to a crash). This architecture provides fault tolerance without relying on name server replication, which is important since a replicated scheme would imply to multiply the number of $\mathrm{LWNS}_{s_{i}}$ by three or four. If the prefix table is inaccessible the multicast method can always be used to resolve a contextual name. If the $\mathrm{LWNS}_{s_{j}}$ responsible for the $o$-ctxt is inaccessible then the probability to find the required information depends on the use of the object: the more often an object is used, the bigger the chance to find the requested information. Most of the time, the resolution will be fast and cheap; costly treatment is only due to failures.

\section{OVERVIEW OF THE DUPLEX ENVIRONMENT}

This Section presents an overview of the DUPLEX collaborative editing environment and points out the naming problem raised by this large scale distributed application. More details about the DUPLEX environment can be found in (Pacull et al., 1994; Pacull, 1995).

Within DUPLEX, the jointly edited document is partitioned into several parts. This partition is dynamic and driven by the authors themselves. Each author works more specifically on one or several parts, but, in order to enhance cooperation, all authors should have direct access to all parts.

Document parts are replicated, to ensure better availability and fault tolerance, and maintained by a distributed kernel. The distributed kernel is shared by all members of the collaboration and provides persistence and availability of the most recent document state. Thus, we call kernel object the set of replicas corresponding to a replicated document part maintained by the kernel.

The co-authors may be scattered over very large distances such as in an international research effort, and interact through the kernel in an asynchronous manner. Basically, as is shown in Figure 5, a user reads an object from the kernel and stores it within a local object. This local object can be modified (edited or decomposed) locally without any connection to the kernel. Finally, the user requests a flush of this local object to the kernel in order to make the new version of the object available to the others co-authors. The concurrency control is provided at the kernel level and several policies (optimistic, pessimistic or hybrid) are offered (Pacull, 1995). The result of the write to the kernel depends on the chosen concurrency control policy.

Thus, a user works locally, disconnected from the kernel, until either (1) a copy of a kernel object is required to continue work, and the user copies it from the kernel, or (2) the user 


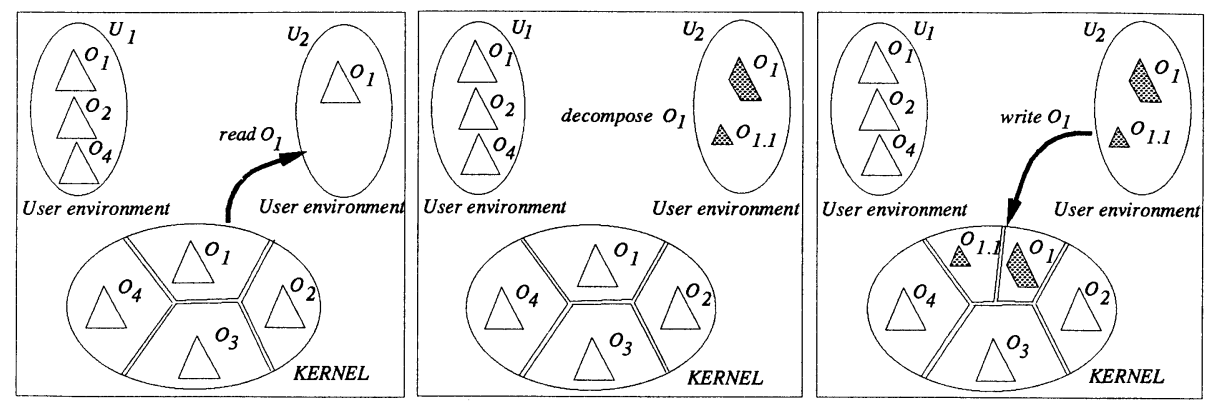

Figure 5 DUPLEX architecture.

considers that a local object version should be shared, and updates the corresponding kernel object. This operating mode allows each user to work locally most of the time and limits the use of communication, that are known to be very costly in large scale.

\subsection{Naming requirement}

The solution we have adopted consists of a kernel distributed over a dozen of sites selected from the domain (local area network) of each collaborating team. Logical objects are partially replicated on these sites, which are chosen for their good communication performance.

Due to the heterogeneous system aspects, several ways to manage replicated objects at the kernel level have to be considered at the same time, implying different patterns for the object reference.

To a document correspond two views: (1) the user's view in which a contextual name corresponds to a local object that belongs to its local file system, and (2) a kernel view, in which each contextual name corresponds to a replicated service that manages the shared object. If the first correspondence (contextual name, local object reference) can be performed statically at the user file system, the second one (contextual name, shared object reference) needs the help of an external name service shared by the community. The set of shared objects is small (usually a few dozen elements) and composed of objects whose names are contextual to the document. Replicas are, however, maintained in heterogeneous file systems with no global naming scheme consistent with the name space of the collaboration. Thus, an important point is related to the way of naming shared objects and of retrieving the data associated to these names. Broadly speaking, the problem is how to embed the small document context into a large distributed system. This problem is delicate and a dedicated name service is required to manage the set of kernel objects distributed over the large heterogeneous network. 


\section{USE OF THE LWNS WITHIN DUPLEX}

The concepts considered for the DUPLEX architecture have some influence on how the LWNS is used. We show in this Section how the LWNS can fulfill the particular requirement of DUPLEX.

\subsection{Architecture of DUPLEX name service}

The LWNS lets the application designer the free choice to decide upon the location of each $\mathrm{LWNS}_{s_{i}}$ and of the LWNS ${ }_{m}$, as well as how the name space is decomposed. The choice made by DUPLEX is to put the LWNS $s_{i}$ close to the users' sites. In other words, if the collaboration involves four research teams whose respective domains are epfl. ch, cornell. edu, unibo. it and inesc.pt, a LWNS is present on one site of each of these domains (e.g. 1 sesun 7 .epf $1 . \mathrm{ch}$, angrboda.cs. cornell. edu, scarpia.dm. unibo. it and pandora. inesc.pt). The LWNS $m$ is located on one single domain, e.g. angrboda. cornell.edu. The format of contextual name that we use ensures that each LWNS $s_{i}$ manages objects created by the users working on the same local area network as $s_{i}$. The pattern of the contextual name in DUPLEX is the following: /doc-name/domain-id/object-name.unique-id. The two fields doc-name and domain-id ensure a correct management of the contextual name at the prefix table level: doc-name operates a segregation on the document name, and domain-id, a logical name defining the domain of a team*, permits to group all the objects created at the same domain, to be managed by the same LWNS $_{s_{i}}$. Finally, object-name. unique-id permits to define a meaningful name for the user that ensures at the same time the unicity of the contextual name in the frame of the collaboration. The user is not disturbed by such complicated contextual names since the graphical interface displays the structure of the document, and each independent object is labeled with the object-name chosen by the object creator. From the user point of view, this is sufficient for naming an object, without any risk of confusion.

Concerning the object references manipulated by DUPLEX, the flexibility of the LWNS permits to consider current implementations of objects, and future extension should not raise any problems. The three primitives of the LWNS, which permit to store, retrieve and delete such information are sufficient, since the validity of the object references is the responsibility of DUPLEX and not the LWNS.

Figure 6 represents several shared objects, corresponding to the document TR-94-10, maintained within the kernel. The boxes on the left hand represent the different local LWNS $_{s_{i}}$ in the domains cornell.edu, epfl.ch and unibo.it. The box on the right hand is the $\mathrm{LWNS}_{m}$ that manages the prefix table. In the present example, the $\mathrm{LWNS}_{m}$ is in charge of two documents (TR-94-10 and IFIP-paper). All contextual names that have as prefix /TR-94-10/lausanne are managed by the LWNS $_{\text {lsesun } 7}$ running on the host lsesun7.epfl. ch. That is not to say that each object created within the domain epfl. ch has to be managed by $\mathrm{LWNS}_{\text {lsesun } 7}$. For instance, $\mathrm{LWNS}_{\text {disun } 10}$ manages contextual names corresponding to IF IP-paper.

The flexibility of the LWNS lets the users choose if the $\mathrm{LWNS}_{s_{j}}$ and the $\mathrm{LWNS}_{m}$ manage information related to one or several documents. This choice is taken when the collaboration begins (for the LWNS $S_{m}$ ), and when a new user enters within the collaboration (the local LWNS $_{s_{j}}$ ). In both cases it is possible to create a new one or use an already existing one.

\footnotetext{
*In the present example, we have chosen the name of the corresponding town.
} 


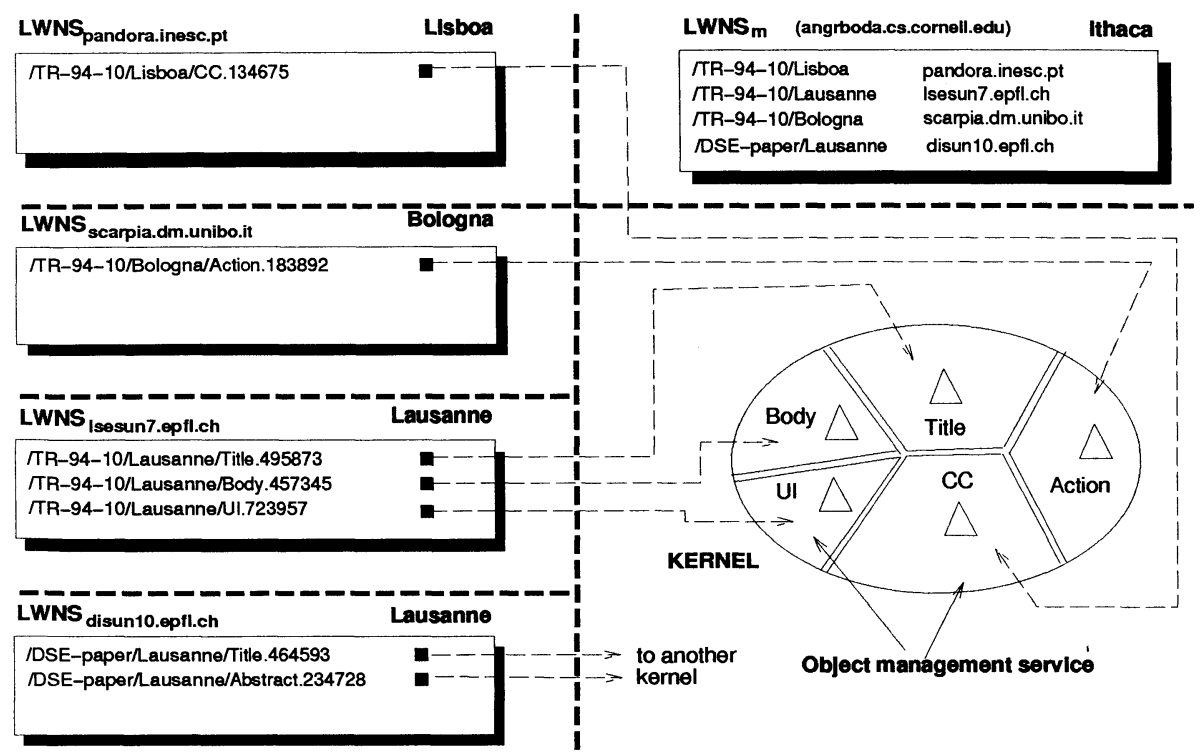

Figure 6 Use of LWNS within DUPLEX.

\subsection{Properties of this architecture}

\section{Efficient object creation}

When considering large scale, the response time is important. Thus, the creation of a new object has to be efficient. This implies that one cannot be delayed by a costly protocol checking if the new contextual name is valid for the registration.

To address this point, we use the fact that with the LWNS, the checking for conflicting names can be disabled. Thus, since (1) the contextual name pattern of DUPLEX permits to generate a unique name, and (2) all the contextual names of objects created at a given site $s_{i}$ are managed by the same LWNS $s_{i}$, the registration can be performed locally. Practically, the creation of the first object by a site requests the LWNS ${ }_{m}$ to modify the prefix table of the LWNS $_{m}$, and all the other creations need not contact neither the LWNS ${ }_{m}$ nor any other $\mathrm{LWNS}_{s_{j}}$. The contextual name is only registered at the $\mathrm{LWNS}_{s_{i}}$ until a user from another domain asks for a remote name resolution. In this case, one of the mechanisms described in Section 3 is used.

\section{Efficient name resolution}

The choice of putting the LWNS $_{s_{i}}$ at the level of the users' site, improves the availability of local resolution, which is one of the goals of the DUPLEX environment.

On one hand, this guarantees that an object created by an user is reachable provided that his/her site is up, and there is no partition isolating the user from the kernel. In this case, both the kernel and the local LWNS are accessible. On the other hand, this is also true for an object already used by a user, due to the cache mechanism. 


\section{Fault-tolerance}

The fault-tolerance for name resolution and new object creation is ensured by the use of the brute force mechanism described in Section 2.

Considering fault-tolerance during the name resolution, we have to distinguish two kinds of failures: (1) the failure of the LWNS $m$ and (2) the failure of the remote LWNS $_{s_{j}}$ that is in charge of the required information*. Due to the brute force mechanism, the first case can be hidden to the user since the required information will eventually be accessible with a longer delay. However, this is not the same for the second case of failure. The probability to resolve a contextual name in such conditions is proportional to the users working with the corresponding object. In fact, the fault-tolerance of the name service is provided by the propagation (in the caches) of information during its use. This guarantees, in particular, that the name resolution, corresponding to important objects, accessed by a large number of people, is always possible. The only problem arises when a contextual name is known only by one LWNS $_{s_{i}}$ and this latter has crashed. In this case, the object is not reachable during the time of the failure.

Even if the user is partitioned from the kernel, he/she is able to create new objects locally and to register them at the local LWNS $_{s_{i}}$. The new objects will be flushed to the kernel when the partition failure is repaired. This enforces the locality of the work for the users.

\section{Low cost name service}

Concerning the cost of the name service, we have to consider two parameters: (1) the cost of a name resolution request, and (2) the amount of information stored at the level of each $\mathrm{LWNS}_{s_{j}}$. For the first point, we have already shown that only the first request is not performed locally, and this first request under normal conditions only requires the consultation of the LWNS $_{m}$ and of one remote LWNS $s_{j}$. For the second point, the information stored by each LWNS $_{s_{j}}$ is minimal since only the contextual names of used objects are maintained either as a shared information or as private information (cache). Moreover, concerning the cost of propagating an information, it is distributed to the requesters and not only paid by the creator. This makes the registration efficient and scalable since it does not depend on the number of $\mathrm{LWNS}_{s_{j}}$ belonging to the cooperation.

\subsection{Discussion}

The implementation of the name service of DUPLEX is not fully fault-tolerant. However, the unique case, (see §Faut-tolerance of Section 5.2), where fault-tolerance is not ensured is not frequent. Moreover, this "partial" fault-tolerance does not rely on any explicit replication. An explicit replication would require much more resources and a more heavy-weight administrative management when a team joins the collaboration. With the solution we have adopted, the new team just needs to launch a LWNS $_{s_{i}}$ and the name service is operational.

The second point to emphasize is that the fault-tolerance provided for the name resolution of a given contextual name is link to the importance of the corresponding object. Thus, the impossibility of resolving a contextual name does not block several teams at the same time since if several teams use an object this latter is known by several LWNS $_{s_{j}}$.

\footnotetext{
*A partition from the kernel has no influence on the name resolution even if, once the name resolution performed,
} the object is not accessible. 
Finally, the low cost of the name service in absence of failures (which is the most frequent case), both for the name resolution and the registration, must be underlined. This is emphasized by the fact that the cache mechanism accelerates the name resolution corresponding to an already accessed object.

\section{RELATED WORK}

Although they are useful in some classes of applications, existing name services present restrictions that are not suitable for use in the specific domain of large scale distributed applications.

First of all, systems that integrate within the same system, a name service that is responsible for the mapping of an object name with the corresponding data, and the protocol required to access these data, could not be used since such systems are not adequate to manipulate general purpose objects (e.g. Ficus (Guy, 1991), Echo (Birrell et al., 1993) manipulate replicated files, and NFS (Sandberg et al., 1985), AFS (Howard et al., 1988), V-system (Cheriton and Mann, 1989), Sprite (Ousterhout et al., 1988) and Amoeba (Tanenbaum et al., 1990) manipulate non replicated files).

Thus, it is necessary to decouple the name service from the access service that manages the access and the storage of the objects. Hence, the name service is responsible for the mapping between a contextual name (the name of the object is the frame of the application) and an object reference. However, the naming service has to be flexible enough to leave the choice of the object reference format to the application builder. For instance, in the case of collaborative application such as DUPLEX, the same object can have two states (activated or desactivated). This requires that the object should be accessible through these two states and the object reference is more complex than a classical service address, since it has to contain the addresses of both the object management service and the activation service. Name services that impose the format of the information associated to a contextual name present a weakness in this matter (e.g. DNS (Terry et al., 1984)).

Moreover, large scale for collaborative applications generally means large distances, rather than a larger number of objects: when people cooperate they share information, so it is not because we have to consider several people that the number of objects will necessarily increase in an exponential manner. However, the probability that the objects and the people are spread over a large network is great, since it depends on the localization of the involved users. Thus, an important point is to provide an efficient name resolution in such a condition. As the number of objects useful for the collaborative application is small compared to the number of objects potentially manipulated by the underlying world wide distributed system, it is inefficient to rely on a global name service such as DCE (Lockhart, 1994). Indeed, the architecture of the underlying network makes the name services of DCE cumbersome to use since it is decomposed into two entities: a cell directory service and a global name service. The first manages the name within its corresponding cell and the second manages the cell name. With a large scale distributed application, the probability that each user is in a different cluster cell is great. Therefore, each resolution will use a costly mechanism even if only few objects are actually of interest for the application.

Another point to raise is that some knowledge about the application can be considered in order to increase the efficiency of the name service. For instance, the collaborative application 
DUPLEX generates a unique contextual name, thus it is not necessary to perform a detection of conflicting names. This ensures a better locality and scalability for the registration since it is not necessary to verify all the entries maintained by the name service at each registration. Most name services (e.g. DCE's (Lockhart, 1994), X500 (CCITT, 1988)) suffer from the impossibility to disable this detection.

The way that the contextual name is used by existing name services is also characterized by a lack of flexibility. Systems like Propero (Neumann, 1992), DCE (Lockhart, 1994), X500 (CCITT, 1988), DNS (Terry et al., 1984) are hierarchical name servers that use the format of the contextual name for deciding which server will manage the name. Here, the server responsible for the management of an object is imposed by the system. In case of a failure of a given server, no other server can provide the same service, and some names might be impossible to register or retrieve during the server failure.

As a result, there is a lack of usable name services in this particular context since none of the existing system could be used for our purpose. The LWNS approach is not to implement a general purpose name service, but to provide a flexible efficient name service that can be adapted for large scale distributed application by providing what is really needed, at a low cost.

\section{CONCLUSION}

This paper has presented a Light Weight Name Service, which is particularly well suited for large scale distributed collaborative applications. We have designed such a name service because no existing system provides the features we need. The LWNS has been used in the design of DUPLEX, a collaborative editing environment, and its use has shown two things.

First, despite of very basic features, the LWNS is well suited for such a large scale application. Verifications are provided at the application level since it is the best way to benefit from the knowledge about the application. No useless work is performed, the application designer pays the price for the guarantees he/she really needs. Moreover, the application designer can adapt the LWNS to his/her requirements. It is not the application that should adapt itself to the name service. More efficient solutions can then be adopted. Second, by the result of its application, it has been proved that the performances were good (Lugeon and Sandoz, 1994) for a name resolution involving sites spread all over the world.

As a conclusion, the LWNS should be considered as a toolkit which permits to create a name service that exactly fits the requirement of a given application. It is not a general purpose name service but it offers a quick name resolution package that is easy to use and customize.

\section{REFERENCES}

Birrell, A., Hisgen, A., Jerian, C., Mann, T., and Swart, G. (1993). The Echo distributed file system. Technical Report 111, Digital Systems Research Center.

CCITT (1988). Recommendation X.500: The directory. CCITT Blue Book, VIII(8).

Cheriton, D. and Mann, T. (1989). Decentralizing a global naming service for improved performance and fault tolerance. ACM Transactions on Computer Systems, 7(2):147-183. 
Comer, D. (1988). Internetworking With TCP/IP: Principles, Protocols, Architecture. Prentice Hall, Stevenage.

Deschrevel, J. P. (1993). The ANSA model for trading and federation. Technical report, APM Cambridge UK.

Guy, R. G. (1991). Ficus: A very large scale reliable distributed file system. Technical Report CSD-910018, University of California, Los Angeles. Ph.D. dissertation.

Howard, J., Kazar, M., Menees, S., Nichols, D., Satyanarayanan, M., Sidebotham, R., and West, M. (1988). Scale and performance in a distributed file system. ACM Transactions on Computer Systems, 6(1):51-81.

Linden, R. V. (1993). ANSA architecture report: The ANSA naming model. Technical report, APM Cambridge UK.

Lockhart, H. (1994). OSF DCE Guide to Developing Distributed Applications. Mc Graw-Hill.

Lugeon, J.-C. and Sandoz, A. (1994). A light weight name service for large scale distributed applications and groupware. Technical Report 94-63, Federal Institute of Technology of Lausanne, Computer Science Departement.

Neumann, B. (1992). The Prospero file system: A global file system based on the virtual system model. In Proceedings of the USENIX File Systems Workshop, pages 13-27.

Ousterhout, J., Cherenson, A., Douglis, F., Nelson, M., and Welch, B. (1988). The Sprite network operating system. IEEE Computer, 21(2):23-36.

Pacull, F. (1995). Concepts et mécanismes pour la mise en oeuvre d' un environnement d'édition coopérative sur un réseau à grande échelle. $\mathrm{PhD}$ thesis, École Polytechnique Fédérale de Lausanne (Federal Institute of Technology of Lausanne).

Pacull, F., Sandoz, A., and Schiper, A. (1994). Duplex: A distributed collaborative editing environment in large scale. In Proceedings of the ACM Conference on Computer-Supported Cooperative Work (CSCW'94), pages 165-173.

Postel, J. and Reynolds, J. (1985). File Transfer Protocol. Network Working Group, RFC 959.

Sandberg, R., Goldberg, D., Kleiman, S., Walsh, D., and Lyon, B. (1985). Design and implementation of the Sun Network File system. In Proceedings of the USENIX Summer Conference, pages 119-130.

Sollins, K. and Clark, D. D. (1988). Distributed name management. In Speth, R., editor, Message Handling Systems, pages 97-115. Elsevier Science Publishers B.V. (North-Holland).

Tanenbaum, A., van Renesse, R., van Staveren, H., Sharp, G., Mullender, S., Jansen, J., and van Rossum, G. (1990). Experience with the Amoeba distributed operating system. Communications of the ACM, 33(12):46-63. 
Terry, D., Painter, M., Riggle, D., and Zhou, S. (1984). The Berkeley Internet Name Domain server. In Proceedings of the USENIX Summer Conference, pages 23-31.

Welch, B. and Ousterhout, J. (1986). Prefix tables: A simple mechanism for locating files in a distributed system. In Proceedings of the 6th International Conference on Distributed Computer Systems, pages 184-189.

\section{BIOGRAPHY}

Jean-Claude Lugeon was born in Geneva in 1964. He obtained a technical degree in Electronic and Computer Science from Engineer School of Geneva, Switzerland, in 1984, and a Master in Computer Science from the Federal Institute of Technology of Lausanne (EPFL) in 1989. He worked in the Operating System Laboratory at the EPFL until end 1994. His research interests are in the area of distributed file service and large scale name service. He is currently system engineer in the Corporate Advanced System Engineering at Landis\&Gyr.

François Pacull received a BSc in Mathematics from the University of Marseille, France, in 1986, and a BSc in Computer Science from the University of Grenoble, France, in 1988. He graduated in Computer Science from the National Polytechnic Institute of Grenoble in 1989. In 1992 he joined the Operating System Laboratory of the Federal Institute of Technology in Lausanne (EPFL), Switzerland, as a research assistant. He received his Ph.D. in Computer Science from the EPFL in 1995. His dissertation was concerned with the impact of large scale distributed systems within the frame of collaborative editing. His research interests are in the area of fault-tolerance and computer supported collaborative work, in particular in the context of large scale distributed systems. 